\title{
Controle Quântico Ótimo: Fundamentos de Controle Ótimo e Extensão para Sistemas Quânticos
}

\author{
Alexandre Coutinho Lisboa ${ }^{1}$ \\ Departamento de Engenharia de Telecomunicações e Controle, Escola Politécnica da Universidade \\ de São Paulo, EPUSP, São Paulo, SP,

\section{José Roberto Castilho Piqueira ${ }^{2}$} \\ Departamento de Engenharia de Telecomunicações e Controle, Escola Politécnica da Universidade \\ de São Paulo, EPUSP, São Paulo, SP.
}

\begin{abstract}
Resumo. Neste artigo, apresentamos fundamentos básicos da Teoria de Controle Ótimo e a extensão dos mesmos para o controle de sistemas quânticos, enfatizando, principalmente, as questões dinâmicas envolvidas, bem como o estabelecimento de funcionais de custo de controle ótimo apropriados ao contexto quântico.
\end{abstract}

Palavras-chave. Controle Quântico, Controle Ótimo, Mecânica Quântica, Informação Quântica.

\section{Introdução}

A Teoria de Controle Ótimo fornece conceitos interessantes e um ferramental poderoso, os quais podem ser aplicados no controle de sistemas quânticos. Em linhas gerais, o Controle Ótimo se debruça sobre o problema de controlar um sistema da forma $\dot{x}(t)=f(x(t), u(t))$ e minimizar, simultaneamente, um funcional do estado $x$ e do controle $u$. Problemas de controle de sistemas quânticos podem ser formulados de forma natural dentro do arcabouço teórico de Controle Ótimo.

Um dos problemas de controle ótimo mais importantes é aquele relacionado com a transferência do estado do sistema para um certo estado-alvo desejado em um tempo mínimo. No contexto quântico, tal problema é de crucial importância. Com efeito, uma das maiores dificuldades de natureza operacional em sistemas quânticos reside no fato de que os mesmos são muito sensíveis à presença do ambiente exterior, o qual freqüentemente destrói as principais características quânticas, que são fundamentais, por exemplo, para a implementação de sistemas de processamento de Informação Quântica, bem como para a

1 alexandrecl@usp.br

2 piqueira@lac.usp.br 
viabilidade da Computação Quântica. Trata-se do problema de Decoerência Quântica [3,4]. Assim, um método que pode ser empregado para contornar a decoerência é obter a transferência desejada de estados no menor tempo possível, de sorte que interação com o ambiente torne-se desprezível.

O tratamento de problemas de controle ótimo vale-se de diferentes ferramentas matemáticas, dependendo se há ou não um limite prescrito para a magnitude do controle. Para controles ilimitados, o problema de controle ótimo de tempo não existe. No entanto, os teoremas de existência de Fillippov garantem que o controle ótimo de transição de estados em tempo mínimo existe sob as hipóteses de Controlabilidade, ou seja, a existência de um controle que realize a meta desejada, funções de controle mensuráveis no sentido de Lebesgue, assumindo valores em um conjunto compacto, e funções que descrevem a dinâmica suaves [1].

De forma rara, e, tipicamente, apenas para casos de dimensionalidade baixa, com simetrias especiais, a solução de um problema de Controle Ótimo pode ser obtida explícita e analiticamente, na forma de uma dada função do tempo. Muito mais freqüentemente, e, em particular para sistemas de maior dimensionalidade, como, por exemplo, em problemas de controle de dinâmicas moleculares, algoritmos numéricos e iterativos são utilizados a fim de se encontrar o controle ótimo apropriado ao sistema de interesse [1,2].

\section{Formulação Geral do Problema de Controle Ótimo}

O problema genérico em Controle Ótimo, com o qual normalmente se depara na Teoria de Controle convencional, diz respeito a encontrar uma função de controle admissível $u^{*}(t)$ que faz com que o sistema dinâmico de interesse siga uma trajetória admissível $x^{*}(t)$, de modo a minimizar uma determinada medida de desempenho ou funcional de custo.

Pode-se formular o problema como se segue. Dado um conjunto $X$ de funções de estado $x(t)$ a valores vetoriais, com $t \in \mathfrak{R}:\left[t_{0}, t_{f}\right], x: \mathfrak{R} \rightarrow \mathfrak{R}^{n}$, e um conjunto $U$ de funções de controle $u(t)$, tais que $u: \mathfrak{R} \rightarrow \mathfrak{R}^{m}$, encontrar as funções $x \in X$ and $u \in U$ para o sistema dinâmico $\dot{x}(t)=f(x(t), u(t)), \operatorname{com} f$ sendo um campo vetorial suave, de modo a minimizar um funcional de custo $J: X \times U \rightarrow \mathfrak{R}$ da forma:

$$
J(u)=h\left(x\left(t_{f}\right), t_{f}\right)+\int_{t_{0}}^{t_{f}} g(x(t), u(t), t) d t
$$

em que, $h$ e $g$ são funções suaves dependendo das variáveis $x(t), u(t)$, e do valor de $x\left(t_{f}\right)$, tais que $h: \mathfrak{R}^{n} \times \mathfrak{R} \rightarrow \mathfrak{R}$ e $g: \mathfrak{R}^{n} \times \mathfrak{R}^{m} \times \mathfrak{R} \rightarrow \mathfrak{R}$, respectivamente. Tais funções são escolhidas judiciosamente tendo em vista o particular problema de controle ótimo que se deseja resolver.

De modo geral, todo problema de Controle Ótimo pode ser formulado como um caso particular do problema geral acima estabelecido.

Consideremos, inicialmente, como caso especial, que desejamos encontrar um dado controle $u$, contínuo por partes, a fim de direcionar o estado $x$ de $\dot{x}(t)=f(x(t), u(t))$, de 
um estado inicial $x_{0}$ para um estado desejado $x_{f}$ em um tempo $T$. Então, $U$ é o conjunto dos de funções de controle $u(t)$, contínuas por partes, definidas no intervalo real $[0, T]$; e, $X$, o conjunto das funções contínuas $x(t)$, definidas no mesmo intervalo, satisfazendo a condição inicial $x(0)=x_{0}$; sendo que o funcional de custo que procuramos minimizar é da forma:

$$
J(u)=\left\|x(T)-x_{f}\right\|^{2}
$$

ou seja, trata-se da minimização da distância entre o estado final e o estado desejado, em um tempo $T$.

Podemos também incorporar ao funcional de custo um termo que leve em consideração a energia gasta (pelo esforço de controle) durante o dado intervalo de tempo. Assim, seja um funcional de custo da seguinte forma:

$$
J(u)=\lambda\left\|x(T)-x_{f}\right\|^{2}+\int_{0}^{T}\|u(t)\|^{2} d t
$$

em que, $\lambda$ é uma constante real e $\lambda>0$. Desta forma, incorporamos uma penalidade no estado final e uma energia como termo para o controle. Pode-se aumentar ou diminuir $\lambda$, se deseja-se ou não empregar mais energia para obter um estado final melhor, ou seja, mais próximo do estado desejado, ou não.

Conforme já antecipado por nós, os funcionais de custo (2.2) e (2.3) consistem em casos especiais do problema geral de Controle Ótimo, estabelecido ao início desta Seção. Com efeito, a expressão (2.1) define o assim chamado "Problema de Bolza" na Teoria de Controle Ótimo, o qual compreende os dois casos particulares, a saber, o "Problema de Mayer" e o "Problema de Lagrange", correspondendo a impor $g(x(t), u(t))$ ou $h\left(x\left(t_{f}\right)\right)$ como sendo zero, respectivamente. Exemplos de problemas de Mayer são problemas de minimização da distância entre o estado final e o estadoalvo desejado, bem como problemas de tempo mínimo, nos quais a meta de controle é levar o estado do sistema a um dado estado-alvo no menor tempo possível. Neste caso, o conjunto de controles admissíveis consiste naqueles que direcionam a dinâmica desta maneira. O problema de Lagrange, por sua vez, descreve uma situação na qual o custo de controle acumula-se com o tempo. Este é o caso, por exemplo, quando se deseja minimizar a energia usada durante a ação de controle e/ou a distância média da trajetória da condição inicial a um dado ponto no espaço de estados. Finalmente, o problema de Bolza, uma vez sendo a combinação de ambos os casos particulares, surge quando há um custo cumulativo que aumenta durante a ação de controle, mas se coloca ênfase também na situação do sistema no tempo final.

\section{Extensão para Sistemas Quânticos}

Em Sistemas de Controle Quântico, podemos considerar como estado $x$ o vetor de estados puro $|\psi\rangle$, a matriz de densidade $\rho$, ou ainda o operador de evolução temporal 
$\hat{U}\left(t, t_{0}\right)$, que satisfaz a equação básica de evolução temporal da Mecânica Quântica, a Equação de Schrödinger, cuja forma controlada pode ser escrita como se segue:

$$
\frac{\partial}{\partial t}|\psi(t)\rangle=-\frac{i}{\hbar} \hat{H}(u(t))|\psi(t)\rangle
$$

na qual se destaca a dependência do Operador Hamiltoniano das funções de controle $u(t)$.

Um funcional genérico de custo, do tipo Bolza, pode ser escrito como:

$$
J(u(t))=h(|\psi(T)\rangle, T)+\int_{0}^{T} g(|\psi(t)\rangle, u(t), t) d t
$$

Alternativamente, pode-se pensar em um funcional de custo expresso em termos do valor esperado das funções $f$ e $g$, para um dado estado quântico $\psi$, vistas como funções dependentes de operadores na Representação de Heisenberg, ou seja, operadores que variam no tempo (com estados quânticos fixos). Destarte, tal funcional de custo se escreveria como:

$$
J(\hat{u}(t))=\langle\psi|h(\hat{x}(T), \hat{u}(T), T)| \psi\rangle+\int_{0}^{T}\langle\psi|g(\hat{x}(t), \hat{u}(t), t)| \psi\rangle d t
$$

sendo, neste caso, considerada uma dependência funcional, por exemplo, dos operadores posição $\hat{x}(t)$ e esforço de controle $\hat{u}(t)$, o qual, fisicamente pode corresponder a um campo elétrico aplicado no sistema, sem ou com dependência da posição, dada pelo operador $\hat{x}(t)$.

Voltando a atenção novamente para a equação de Schrödinger controlada (3.1) e para o funcional de custo (3.2), e considerando-se o caso de sistemas quânticos discretos, ou seja, com um espectro finito de autovalores, tanto o estado $|\psi(t)\rangle$, como o operador $-\frac{i}{\hbar} \hat{H}(u(t))$, dado no caso discreto por uma matriz hermiteana, são quantidades complexas. Para expressar o problema em termos de quantidades reais apenas, pode-se escrever:

$$
\begin{gathered}
|\psi\rangle=\left|\psi_{R}\right\rangle+i\left|\psi_{I}\right\rangle \\
-i \hat{H}(u)=R(u)+i I(u)
\end{gathered}
$$

de modo a separar, nas partes real e complexa, o estado e o operador hamitoniano, respectivamente. Assim, $\left|\psi_{R}\right\rangle$ e $\left|\psi_{I}\right\rangle$ são agora vetores reais de dimensão $n$, e $R(u)$ e $I(u)$, funções matriciais $n \times n$ de $u(t)$. Substituindo-se as equações (3.4) e (3.5) em (3.1), e separando-se as partes real e imaginária, obtemos as duas equações diferenciais reais: 


$$
\begin{aligned}
& \frac{d}{d t} \psi_{R}=R(u) \psi_{R}-I(u) \psi_{I} \\
& \frac{d}{d t} \psi_{I}=I(u) \psi_{R}+R(u) \psi_{I}
\end{aligned}
$$

Definindo-se:

$$
\begin{gathered}
x \equiv\left[\begin{array}{ll}
\psi_{R}^{T} & \psi_{I}^{T}
\end{array}\right]^{T} \\
\tilde{H}(u) \equiv\left(\begin{array}{cc}
R(u) & -I(u) \\
I(u) & R(u)
\end{array}\right)
\end{gathered}
$$

podemos escrever a equação diferencial que descreve a dinâmica do sistema, envolvendo apenas quantidades reais, como:

$$
\dot{x}=\tilde{H}(u) x
$$

de modo a expressar o sistema em uma linguagem mais próxima daquela empregada comumente em Sistemas Dinâmicos. Uma simples mudança de escala permite restaurar a constante fundamental da Mecânica Quântica, com dimensão de ação (energia $\times$ tempo), $\hbar$. Ademais, $\tilde{H}(u)$ resulta anti-simétrico para todo $u(t)$ [1].

$\mathrm{O}$ funcional de custo pode ser reescrito na forma:

$$
J(u(t))=\tilde{h}(x(T), T)+\int_{0}^{T} \tilde{g}(x(t), u(t), t) d t
$$

para funções apropriadas $\tilde{h}$ e $\tilde{g}$.

A título de exemplos, em aplicações de controle molecular, uma escolha comum de funcional é dada por:

$$
J(u(t))=k \int_{0}^{T} u^{2}(t) d t
$$

com uma constante real, $k>0$, a qual mede a energia do campo elétrico aplicado no intervalo $[0, T]$, como esforço de controle $u(t)$.

Outra possibilidade é escolher um custo do tipo: 


$$
J(u(t))=k \int_{0}^{T}\left(\frac{d u(t)}{d t}\right)^{2} d t
$$

o qual filtra as componentes de alta freqüência do campo de controle; sendo $k$ uma constante real, $k>0$.

Quando a ênfase é colocada em um determinado estado final, pode-se minimizar um funcional de custo (tipo Bolza) na forma:

$$
J(u(t))=\lambda\left\|x(T)-x_{F}\right\|^{2}+\int_{0}^{T}\|u(t)\|^{2} d t
$$

ou, ainda, na forma:

$$
\frac{1}{2}\langle O\rangle_{\psi}+\frac{k}{2} \int_{0}^{T} u^{2}(t) d t=\frac{1}{2} \psi^{\dagger} O \psi+\frac{k}{2} \int_{0}^{T} u^{2}(t) d t
$$

com $k>0$; no qual, $O$ é uma matriz hermiteana e negativa-definida [1]. Por exemplo, se $\psi_{F}$ é o estado desejado, pode-se escolher $O=-\psi_{F} \psi_{F}^{\dagger}$.

\section{Conclusões}

Este artigo teve como meta apresentar a Teoria de Controle Quântico Ótimo como extensão da Teoria Clássica de Controle Ótimo, discutindo os aspectos físicos e matemáticos envolvidos nesta transição. Questões fenomenológicas, computacionais e perspectivas de aplicações tecnológicas são também abordadas.

A partir da Dinâmica Quântica, apresentamos a equação de Schrödinger controlada, bem como estabelecemos funcionais de custo de controle ótimo adequados ao domínio quântico, a serem vistos como uma generalização dos funcionais de controle ótimo clássico. Em seguida, para sistemas quânticos de dimensão finita, ou seja, dotados de um espectro finito de autovalores, foi possível escrever os vetores correspondentes aos estados quânticos, bem como o operador Hamiltoniano do sistema, em forma matricial, valendo-se de quantidades reais apenas. Para tanto, foi necessário separar os estados e o operador Hamiltoniano em suas partes real e imaginária, dobrando-se, com isso, a dimensão do sistema resultante. Como ganho, não obstante, o sistema, agora, a valores reais, é análogo aos sistemas dinâmicos comumente encontrados, de sorte que o ferramental de Controle Ótimo, tanto teórico, quanto numérico ou computacional, pode ser prontamente empregado para a obtenção de soluções.

Em um artigo subseqüente, discutiremos as condições básicas de optimalidade, a saber, o Princípio do Máximo de Pontryagin (PMP), e como aplicá-lo a problemas de Controle Quântico Ótimo. 


\section{Referências}

[1] D. D'Alessandro, Introduction to Quantum Dynamics and Control. Boca Raton, FL: Chapman\&Hall / CRC, (2007).

[2] V. F. Krotov, Управление Квантовыми Системами и Некоторые Идеи Теории Оптимального Управления. Автоматика и телематика, № 3, 15-23, (2009).

[3] M. A. Nielsen, I. L. Chuang, Quantum Computation and Quantum Information, Cambridge, UK: Cambridge University Press, (2000).

[4] M. A. Schlosshauer, Decoherence and the Quantum-to-Classical Transition, NY: Springer Verlag, (The Frontiers Collection), (2008). 